\title{
Variance analysis for model updating with a finite element based subspace fitting approach
}

\author{
Guillaume Gautier $^{\mathrm{a}, \mathrm{b}, *}$, Laurent Mevel ${ }^{\mathrm{b}}$, Jean-Mathieu Mencik ${ }^{\mathrm{c}}$, Roger Serra $^{\mathrm{c}}$, Michael Döhler ${ }^{\mathrm{b}}$ \\ ${ }^{a}$ CEA-Tech Pays-de-la-Loire, Technocampus Océan, 5 rue de l'Halbrane, 44340 Bouguenais, France \\ ${ }^{b}$ Inria / IFSTTAR, I4S, Campus de Beaulieu, 35042 Rennes, France \\ ${ }^{c}$ INSA Centre Val de Loire, Université François Rabelais de Tours, LMR EA 2640, Campus de Blois, 3 rue de la chocolaterie, CS 23410, \\ 41034 Blois Cedex, France
}

\begin{abstract}
Recently, a subspace fitting approach has been proposed for vibration-based finite element model updating. The approach makes use of subspace-based system identification, where the extended observability matrix is estimated from vibration measurements. Finite element model updating is performed by correlating the model-based observability matrix with the estimated one, by using a single set of experimental data. Hence, the updated finite element model only reflects this single test case. However, estimates from vibration measurements are inherently exposed to uncertainty due to unknown excitation, measurement noise and finite data length. In this paper, a covariance estimation procedure for the updated model parameters is proposed, which propagates the data-related covariance to the updated model parameters by considering a first-order sensitivity analysis. In particular, this propagation is performed through each iteration step of the updating minimization problem, by taking into account the covariance between the updated parameters and the data-related quantities. Simulated vibration signals are used to demonstrate the accuracy and practicability of the derived expressions. Furthermore, an application is shown on experimental data of a beam.
\end{abstract}

Keywords: Stochastic system identification, Subspace fitting, Uncertainty bounds, Finite element model

\section{Introduction}

Linear system identification methods are of interest in mechanical engineering for modal analysis. Using outputonly vibration measurements from structures, Operational Modal Analysis (OMA) has been successfully used as a complementary technique to the traditional Experimental Modal Analysis (EMA) methods [1-3]. With methods originating from stochastic system realization theory for linear systems, estimates of the modal parameters of interest (natural frequencies, damping ratios and observed mode shapes) can be obtained from vibration data. Among these methods, the stochastic subspace identification (SSI) techniques [4, 5] identify the system matrices of a state-space model, from which the modal parameters are retrieved. Subspace methods are well-suited for the vibration analysis

*Corresponding author; Email address: guillaume.gautier@ifsttar.fr ; Phone number: +33240845902 
of structures in operation, which is due to the fact that they have excellent theoretical and computational properties, for instance numerical efficiency and robustness, see e.g. [6, 7].

For any system identification method, the estimated parameters are afflicted with variance errors due to finite data length, unknown excitation and measurement noise. The variance of the modal parameter estimates is a most relevant information for assessing their accuracy. It depends on the chosen system identification algorithm. A practical approach for the variance estimation of modal parameters was developed in [8], where an estimated covariance on the measurements is propagated to the desired parameters by considering a sensitivity analysis. The required sensitivities are derived analytically through first-order perturbation theory, from the data to the identified parameters, and are then computed using the system identification estimates. In [9], the covariance computation scheme for the covariancedriven subspace method (SSI-cov) has been developed, and in [10] a fast and memory efficient implementation of the covariance computation for SSI-cov has been proposed.

The identification of modal parameters or, more generally, the system identification results of a structure in operation find an important application in the calibration of a numerical model of the investigated structure. Finite element (FE) models are used e.g. to verify design specifications, to assess stress fields in structures, to predict vibration levels under prescribed harmonic excitations, to detect abnormal structural behavior in the context of structural health monitoring [11, 12], and so on. With model updating techniques, the parameters of the FE model are calibrated such that some model properties are close to the truly observed structural properties. Vibration-based FE model updating techniques $[13,14]$ identify model parameters by minimizing a cost function involving the identified and model-based modal parameters (or derived variables thereof). The involved experimental data are subject to uncertainties. In a broad sense, these uncertainties can be classified into two categories of aleatory (irreducible) and epistemic (reducible) uncertainties [15]. Aleatory uncertainty may result from geometric dimension variability due to manufacturing tolerances or inherent variability of materials such as concrete, while epistemic uncertainty is caused by lack of knowledge (e.g. due to finite number of data samples, undefined measurement noises, unknown excitations, and so on). These uncertainties can be considered in two ways in model updating. First, the uncertainty of the updated parameters can be evaluated based on the uncertainty of the experimental data. Second, the uncertainty of the experimental data can be taken into account in stochastic updating techniques as suggested in [12]. In this paper, we consider the first way.

While many FE updating methods analyze the impact of aleatory structural uncertainties on the updated parameters (see for instance [16]), we consider the problem of evaluating the uncertainty of the updated FE parameters that result from the uncertainty when estimating parameters from vibration measurements. This statistical uncertainty falls into the category of epistemic uncertainties since it reduces as the number of measurements increases. We consider a recently proposed subspace fitting (SF) approach for FE model updating [17], which is a deterministic approach closely linked to subspace identification [18]. In this framework, the model parameters of a coarse FE mesh are updated in a minimization problem that consists in correlating a FE-based extended observability matrix with an experimental one that is identified from SSI-cov. Since the experimental observability matrix is estimated 
from measured vibration data, it is afflicted with uncertainty. In this paper, we propose a covariance analysis of the structural parameters obtained from the SF approach taking into account this uncertainty. The identified observability matrix and its covariance are estimated with SSI-cov $[9,10]$ and propagated to the updated FE parameters by means of a sensitivity analysis. The expressions of the covariances of the FE parameters are an original contribution of the present paper. In particular, we show how the covariance originating from the vibration data is propagated through the iterations of the minimization problem for the solution of the FE parameters, taking into account the covariance between the parameter estimate in each iteration and the data-related quantities. The derived expressions are validated through Monte Carlo simulations. Notice that the covariance estimation from multiple datasets as in Monte Carlo simulations is computationally much more demanding [19], compared to the covariance estimation from a single dataset as in the proposed approach. Also, an application of the approach on a lab experiment is reported.

The paper is organized as follows. In Section 2, the theoretical framework of the FE-based SF method is presented. The covariance expressions of the updated FE model parameters are derived in Section 3. In Section 4, the covariance estimation procedure is illustrated regarding the vibration data issued from a numerical model of a beam. Results are validated with standard deviations obtained from Monte-Carlo simulations. Finally, in Section 5, the proposed method is applied to model updating using experimental data of a beam.

\section{SF method for model updating}

Model updating has the purpose to calibrate the parameters of a FE model such that some model properties are close to the truly observed structural properties. In the proposed SF method, the considered quantity for calibration is the extended observability matrix $O$ of the underlying linear system. The main idea is to correlate - in a least squares sense - the matrix $\hat{O}$ obtained from experimental data with the matrix $O^{h}\left(\boldsymbol{\theta}^{h}\right)$ issued from a FE model of the structure, where $\boldsymbol{\theta}^{h} \in \mathbb{R}^{n_{h}}$ is the vector of structural parameters of the FE model to be updated.

In this section, the subspace identification method is introduced to obtain $\hat{O}$ from experimental data, and the SF method, based on $\hat{O}$ and $O^{h}\left(\boldsymbol{\theta}^{h}\right)$ for updating the vector of parameters $\boldsymbol{\theta}^{h}$ is presented.

\subsection{Stochastic subspace identification}

The vibration behavior of a linear elastic structure, which is observed at some sensor positions, can be described through the following equations [1]

$$
\left\{\begin{aligned}
\mathbf{M} \ddot{\mathbf{q}}(t)+\gamma \dot{\mathbf{q}}(t)+\mathbf{K q}(t) & =\mathbf{v}(t), \\
\mathbf{y}(t) & =\mathbf{H}_{d} \mathbf{q}(t)+\mathbf{H}_{v} \dot{\mathbf{q}}(t)+\mathbf{H}_{d} \ddot{\mathbf{q}}(t)+\mathbf{w}(t),
\end{aligned}\right.
$$

where $\mathbf{M} \in \mathbb{R}^{n \times n}, \mathbf{K} \in \mathbb{R}^{n \times n}$ and $\gamma \in \mathbb{R}^{n \times n}$ are, respectively, the mass, stiffness and viscous damping matrices, $t$ denotes continuous time, $\mathbf{q} \in \mathbb{R}^{n}$ is the vector of displacements, and $n$ is the number of degrees of freedom (DOFs). Boolean matrices $\mathbf{H}_{d}, \mathbf{H}_{v}$ and $\mathbf{H}_{a} \in \mathbb{R}^{r \times n}$ localize the DOFs at which displacements, velocities and accelerations are measured in 
the vector $\mathbf{y} \in \mathbb{R}^{r}$. Finally, $\mathbf{v} \in \mathbb{R}^{n}$ and $\mathbf{w} \in \mathbb{R}^{r}$ are white noise vectors of unmeasured excitation forces and measurement noise, respectively.

Sampling system (1) at discrete time instants $t=k \tau$, where $\tau$ is the sampling rate, leads to the discrete-time linear time-invariant (LTI) state-space model

$$
\left\{\begin{aligned}
\mathbf{x}_{k+1} & =\mathbf{A} \mathbf{x}_{k}+\mathbf{v}_{k} \\
\mathbf{y}_{k} & =\mathbf{C} \mathbf{x}_{k}+\mathbf{w}_{k},
\end{aligned}\right.
$$

where $\mathbf{x}_{k}=\left[\mathbf{q}(k \tau)^{T} \dot{\mathbf{q}}(k \tau)^{T}\right]^{T} \in \mathbb{R}^{2 n}$ is the state vector, $\mathbf{y}_{k} \in \mathbb{R}^{r}$ is the vector of measurement outputs. Also, $\mathbf{A} \in \mathbb{R}^{2 n \times 2 n}$ and $\mathbf{C} \in \mathbb{R}^{r \times 2 n}$ are state transition and output matrices, respectively, expressed by

$$
\mathbf{A}=\exp \left(\left[\begin{array}{cc}
\mathbf{0} & \mathbf{I}_{n} \\
-\mathbf{M}^{-1} \mathbf{K} & -\mathbf{M}^{-1} \gamma
\end{array}\right] \tau\right) \quad, \quad \mathbf{C}=\left[\begin{array}{lll}
\mathbf{H}_{d}-\mathbf{H}_{a} \mathbf{M}^{-1} \mathbf{K} & \mid & \mathbf{H}_{v}-\mathbf{H}_{a} \mathbf{M}^{-1} \gamma
\end{array}\right] .
$$

The vectors $\mathbf{v}_{k} \in \mathbb{R}^{2 n}$ and $\mathbf{w}_{k} \in \mathbb{R}^{r}$ are zero-mean white noise, i.e., $\mathbf{E}\left[\mathbf{v}_{k}\right]=0$ and $\mathbf{E}\left[\mathbf{w}_{k}\right]=0$, with covariance matrices [4]

$$
\mathbf{E}\left[\left(\begin{array}{c}
\mathbf{w}_{p} \\
\mathbf{v}_{p}
\end{array}\right)\left(\begin{array}{ll}
\mathbf{w}_{q} & \mathbf{v}_{q}
\end{array}\right)\right]=\left(\begin{array}{cc}
\mathbf{Q} & \mathbf{S} \\
\mathbf{S}^{T} & \mathbf{R}
\end{array}\right) \delta_{p q},
$$

where $\delta_{p q}$ is the Kronecker delta. The stochastic process (2) is assumed to be stationary, i.e., $\mathbf{E}\left[\mathbf{x}_{k+1} \mathbf{x}_{k}^{T}\right]=\Sigma$ where $\Sigma$ $\in \mathbb{R}^{2 n \times 2 n}$ does not depend on time $k$, with zero mean, i.e., $\mathbf{E}\left[\mathbf{x}_{k}\right]=0$. Also, $\mathbf{v}_{k}$ and $\mathbf{w}_{k}$ are assumed to be independent on the actual state, i.e., $\mathbf{E}\left[\mathbf{x}_{k} \mathbf{v}_{k}^{T}\right]=0$ and $\mathbf{E}\left[\mathbf{x}_{k} \mathbf{w}_{k}^{T}\right]=0$.

Define the output covariance matrix $\mathcal{R}_{i} \in \mathbb{R}^{r \times r}$ as

$$
\mathcal{R}_{i}=\mathbf{E}\left[\mathbf{y}_{k+i} \mathbf{y}_{k}^{T}\right],
$$

and the state-output covariance matrix $\mathbf{G} \in \mathbb{R}^{2 n \times r}$ as

$$
\mathbf{G}=\mathbf{E}\left[\mathbf{x}_{k+1} \mathbf{y}_{k}^{T}\right]
$$

The output covariance matrices $\mathcal{R}_{i}$ can be stacked into a block Hankel matrix $\mathcal{H} \in \mathbb{R}^{(p+1) r \times q r}$, where $p$ and $q$ are chosen such that $\min (p r, q r) \geq 2 n$, as follows

$$
\mathcal{H}=\left[\begin{array}{cccc}
\mathcal{R}_{1} & \mathcal{R}_{2} & \ldots & \mathcal{R}_{q} \\
\mathcal{R}_{2} & \mathcal{R}_{3} & \ldots & \mathcal{R}_{q+1} \\
\vdots & \vdots & \ddots & \vdots \\
\mathcal{R}_{p+1} & \mathcal{R}_{p+2} & \ldots & \mathcal{R}_{p+q}
\end{array}\right]
$$

The Hankel matrix $\mathcal{H}$ has the factorization property [20]

$$
\mathcal{H}=O C,
$$


where $O \in \mathbb{R}^{(p+1) r \times 2 n}$ and $C \in \mathbb{R}^{2 n \times q r}$ are the extended observability matrix and controllability matrix, respectively, which are given by

$$
O=\left[\begin{array}{c}
\mathbf{C} \\
\mathbf{C A} \\
\vdots \\
\mathbf{C A}^{p}
\end{array}\right], \quad C=\left[\begin{array}{llll}
\mathbf{G} & \mathbf{A G} & \ldots & \mathbf{A}^{q-1} \mathbf{G}
\end{array}\right]
$$

Notice that the matrix $O$ is full column rank [4, 17]. Within the SSI framework [5], an estimate $\hat{\mathcal{H}}$ of the output Hankel matrix $\mathcal{H}$ can be built from $N+p+q$ measurements as

$$
\hat{\mathcal{H}}=\frac{1}{N} \mathcal{Y}^{+}\left(\boldsymbol{y}^{-}\right)^{T},
$$

where

$$
\boldsymbol{y}^{-}=\left[\begin{array}{cccc}
\mathbf{y}_{q} & \mathbf{y}_{q+1} & \ldots & \mathbf{y}_{N+q-1} \\
\mathbf{y}_{q-1} & \mathbf{y}_{q} & \ldots & \mathbf{y}_{N+q-2} \\
\vdots & \vdots & \ddots & \vdots \\
\mathbf{y}_{1} & \mathbf{y}_{2} & \ldots & \mathbf{y}_{N}
\end{array}\right], \quad \boldsymbol{y}^{+}=\left[\begin{array}{cccc}
\mathbf{y}_{q+1} & \mathbf{y}_{q+2} & \ldots & \mathbf{y}_{N+q} \\
\mathbf{y}_{q+2} & \mathbf{y}_{q+3} & \ldots & \mathbf{y}_{N+q+1} \\
\vdots & \vdots & \ddots & \vdots \\
\mathbf{y}_{q+p+1} & \mathbf{y}_{q+p+2} & \ldots & \mathbf{y}_{N+p+q}
\end{array}\right]
$$

From (8), it can be readily proved that $\mathcal{H}$ and $O$ share the same column space. Hence, an estimate $\hat{O}$ of the extended observability matrix can be obtained from a Singular Value Decomposition (SVD) of $\hat{\mathcal{H}}$ and its truncation at the order $2 n$

$$
\hat{\mathcal{H}}=\mathbf{U} \boldsymbol{\Delta} \mathbf{V}^{T}=\left[\begin{array}{ll}
\mathbf{U}_{1} & \mathbf{U}_{0}
\end{array}\right]\left[\begin{array}{cc}
\boldsymbol{\Delta}_{1} & \mathbf{0} \\
\mathbf{0} & \boldsymbol{\Delta}_{0}
\end{array}\right]\left[\begin{array}{l}
\mathbf{V}_{1}^{T} \\
\mathbf{V}_{0}^{T}
\end{array}\right] \approx \mathbf{U}_{1} \boldsymbol{\Delta}_{1} \mathbf{V}_{1}^{T},
$$

and thus

$$
\hat{O}=\mathbf{U}_{1}
$$

where $\boldsymbol{\Delta}_{1}$ is the diagonal matrix of the $2 n$ dominant singular values of $\hat{\mathcal{H}}$, and $\mathbf{U}_{1} \in \mathbb{R}^{(p+1) r \times 2 n}$ is the related matrix of left singular vectors. While estimates $(\hat{\mathbf{C}}, \hat{\mathbf{A}})$ of the system matrices and subsequently the modal parameters are obtained from $\hat{O}$ in subspace identification, the SF approach requires only the matrix $\hat{O}$ in the following.

Remark 1. The LTI state-space system (2) is defined up to a change of basis. Hence, for any invertible transformation matrix $\mathbf{T}$, the matrix systems defined by $(\mathbf{C}, \mathbf{A})$ or $\left(\mathbf{C T}, \mathbf{T}^{-1} \mathbf{A T}\right)$ are equivalent and share the same modal parameters (eigenfrequencies, observed mode shapes and modal dampings), with respective observability matrices $O$ or $O \mathbf{T}$. Thus, any of these state-space systems may be considered. Notice however that the true transformation matrix $\mathbf{T}-$ i.e., the one which is considered to express the estimate of $O$ in (13) in the basis corresponding to (3) - is unknown. In other words, there exists an unknown matrix $\mathbf{T}$ such that $\hat{O} \mathbf{T}$ is an estimate of $O$ in (9). 


\subsection{FE-based SF method}

In many applications, a numerical FE model is available that approximates the vibration behavior of a structure whose dynamic equilibrium equation is provided by (1)-(2). This model yields an analogous equation of motion

$$
\left\{\begin{aligned}
\mathbf{M}^{h} \ddot{\mathbf{q}}^{h}(t)+\gamma^{h} \dot{\mathbf{q}}^{h}(t)+\mathbf{K}^{h} \mathbf{q}^{h}(t) & =\mathbf{v}^{h}(t) \\
\mathbf{y}(t) & =\mathbf{H}_{d}^{h} \mathbf{q}^{h}(t)+\mathbf{H}_{v}^{h} \dot{\mathbf{q}}^{h}(t)+\mathbf{H}_{d}^{h} \ddot{\mathbf{q}}^{h}(t)+\mathbf{w}^{h}(t),
\end{aligned}\right.
$$

where $\mathbf{M}^{h} \in \mathbb{R}^{n \times n}, \mathbf{K}^{h} \in \mathbb{R}^{n \times n}$ and $\boldsymbol{\gamma}^{h} \in \mathbb{R}^{n \times n}$ are, respectively, the mass, stiffness and viscous damping matrices which are issued from the FE modeling of the structure, and the other variables being analogously defined as in (1). The matrices $\mathbf{M}^{h}, \mathbf{K}^{h}$ and $\boldsymbol{\gamma}^{h}$ depend on a vector $\boldsymbol{\theta}^{h}=\left[\begin{array}{lll}\theta_{1}^{h} & \theta_{2}^{h} \cdots & \theta_{n_{h}}^{h}\end{array}\right]^{T} \in \mathbb{R}^{n_{h}}$, which contains the structural parameters that will be updated.

Similarly as in the previous section, sampling (14) at rate $\tau$ leads to a discrete-time state-space model as in (2) with the system matrices

$$
\mathbf{A}^{h}=\exp \left(\left[\begin{array}{cc}
\mathbf{0} & \mathbf{I}_{n} \\
-\left(\mathbf{M}^{h}\right)^{-1} \mathbf{K}^{h} & -\left(\mathbf{M}^{h}\right)^{-1} \boldsymbol{\gamma}^{h}
\end{array}\right] \tau\right), \quad \mathbf{C}^{h}=\left[\begin{array}{ll}
\mathbf{H}_{d}^{h}-\mathbf{H}_{a}^{h}\left(\mathbf{M}^{h}\right)^{-1} \mathbf{K}^{h} & \mid
\end{array} \quad \mathbf{H}_{v}^{h}-\mathbf{H}_{a}^{h}\left(\mathbf{M}^{h}\right)^{-1} \boldsymbol{\gamma}^{h}\right],
$$

which depend on the parameters $\left\{\theta_{j}^{h}\right\}_{j}$. Also, the related extended observability matrix is expressed by

$$
O^{h}\left(\boldsymbol{\theta}^{h}\right)=\left[\begin{array}{c}
\mathbf{C}^{h} \\
\mathbf{C}^{h} \mathbf{A}^{h} \\
\vdots \\
\mathbf{C}^{h}\left(\mathbf{A}^{h}\right)^{p}
\end{array}\right]
$$

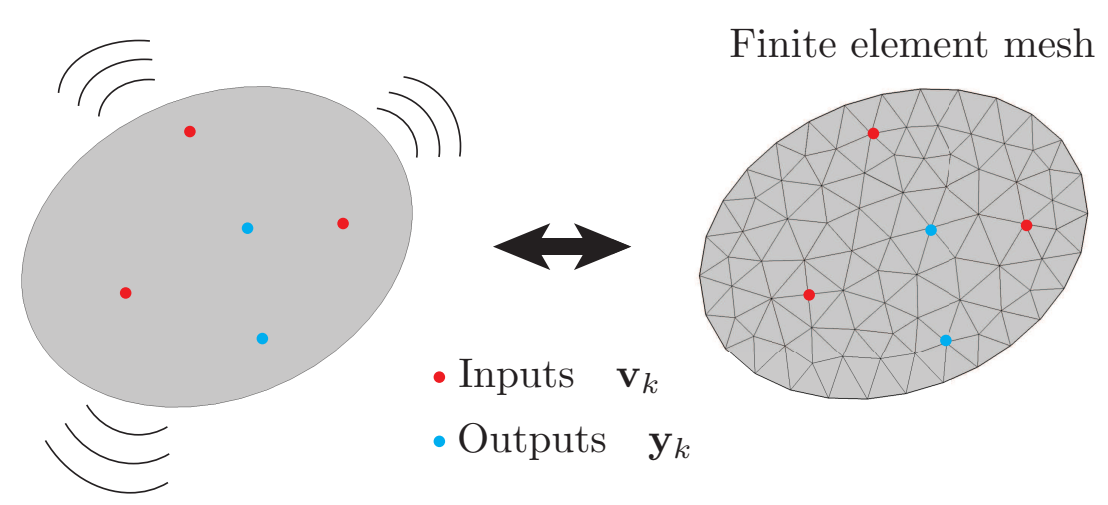

Figure 1: Vibrating structure (left) and corresponding FE mesh discretization (right).

When the assumed FE model fits the experimental data perfectly, the extended observability matrices $O^{h}\left(\boldsymbol{\theta}^{h}\right)$ issued from the FE model and $\hat{O}$ estimated from experimental data are equal up to a change of basis (see Remark 1), i.e. $O^{h}\left(\boldsymbol{\theta}^{h}\right)=\hat{O} \mathbf{T}$ for some matrix $\mathbf{T}$. Then, both matrices share the same column space. However, this is hardly the 
case in practice and the vector of parameters $\boldsymbol{\theta}^{h}$ needs to be adjusted to satisfy this condition. The key idea behind SF is to adjust the respective subspace defined by $O^{h}\left(\boldsymbol{\theta}^{h}\right)$ such that

$$
\boldsymbol{\theta}^{h}=\operatorname{argmin}\left\|O^{h}\left(\boldsymbol{\theta}^{h}\right)-\hat{O} \mathbf{T}\right\|_{F}^{2},
$$

where $\|\cdot\|_{F}$ denotes the Frobenius norm.

Assume that the matrix $O^{h}$ depends on a few parameters $\boldsymbol{\theta}^{h}$. This leads to a minimization problem whose resolution does not require excessive computational times.

The first step in solving this minimization problem is to express the unknown matrix $\mathbf{T}$. It is easy to see that

$$
\mathbf{T}=\hat{O}^{\dagger} O^{h}\left(\boldsymbol{\theta}^{h}\right)
$$

is the minimum-norm solution of the linear least squares problem (17) for fixed $\boldsymbol{\theta}^{h}$, where $\hat{O}^{\dagger}$ is the Moore-Penrose pseudoinverse of $\hat{O}$. By replacing the expression of $\mathbf{T}$ in the original problem (17), the minimization problem takes the following form [21]

$$
\boldsymbol{\theta}^{h}=\operatorname{argmin}\left\|\left(\mathbf{I}_{(p+1) r}-\hat{O} \hat{O}^{\dagger}\right) O^{h}\left(\boldsymbol{\theta}^{h}\right)\right\|_{F}^{2}
$$

Using vector notations with the column stacking vectorization operator vec $\{\cdot\}$, the minimization problem turns out to be

$$
\boldsymbol{\theta}^{h}=\operatorname{argmin}\|\mathbf{r}\|_{2}^{2}
$$

where $\mathbf{r} \in \mathbb{R}^{2(p+1) r n}$ has the meaning of a residual vector, defined as

$$
\mathbf{r}=\left[\mathbf{I}_{2 n} \otimes\left(\mathbf{I}_{(p+1) r}-\hat{O} \hat{O}^{\dagger}\right)\right] \operatorname{vec}\left\{O^{h}\left(\boldsymbol{\theta}^{h}\right)\right\},
$$

and $\|\cdot\|_{2}$ is the Euclidean vector norm. One of the most popular and effective algorithms for solving least squares problems like (20) is the Gauss-Newton method [22]. The method is based on a second-order expansion of the objective function $\|\mathbf{r}\|_{2}^{2}$ about some approximated values of the parameters $\boldsymbol{\theta}^{h}$, and iteration steps so as to find the local extrema of $\|\mathbf{r}\|_{2}^{2}$. Assuming an initial deterministic parameter value $\boldsymbol{\theta}_{0}^{h}$, the $k$-th iteration of the Gauss-Newton algorithm can be written as

$$
\boldsymbol{\theta}_{k}^{h}=\boldsymbol{\theta}_{k-1}^{h}-\mathcal{J}_{\mathbf{r}_{k}}^{\dagger} \mathbf{r}_{k}
$$

for $k \geq 1$, where $\boldsymbol{\theta}_{k}^{h}=\left[\theta_{1, k}^{h} \cdots \theta_{n_{h}, k}^{h}\right]^{T}$ and $\boldsymbol{\theta}_{k-1}^{h}=\left[\theta_{1, k-1}^{h} \cdots \theta_{n_{h}, k-1}^{h}\right]^{T}$ are the vectors of structural parameters identified at iterations $k$ and $k-1$ of the Gauss-Newton algorithm, respectively. Also, $\mathbf{r}_{k}$ is the residual vector at iteration $k$

$$
\mathbf{r}_{k}=\left[\mathbf{I}_{2 n} \otimes\left(\mathbf{I}_{(p+1) r}-\hat{O} \hat{O}^{\dagger}\right)\right] \operatorname{vec}\left\{O^{h}\left(\boldsymbol{\theta}_{k-1}^{h}\right)\right\},
$$

$\mathcal{J}_{\mathbf{r}_{k}}^{\dagger}$ is the Moore-Penrose pseudoinverse of the Jacobian matrix $\mathcal{J}_{\mathbf{r}_{k}} \in \mathbb{R}^{2(p+1) r n \times n_{h}}$, namely the derivative of $\mathbf{r}_{k}$ with respect to the approximated values of $\boldsymbol{\theta}^{h}$

$$
\mathcal{J}_{\mathbf{r}_{k}}=\left[\mathbf{I}_{2 n} \otimes\left(\mathbf{I}_{(p+1) r}-\hat{O} \hat{O}^{\dagger}\right)\right] \mathcal{J}_{O^{h}, \boldsymbol{\theta}_{k-1}^{h}} .
$$


In (22), $\mathcal{J}_{O^{h}, \theta_{k-1}^{h}}$ is given by

$$
\mathcal{J}_{O^{h}, \boldsymbol{\theta}_{k-1}^{h}}=\left.\left[\begin{array}{lll}
\frac{\partial \mathrm{vec}\left\{O^{h}\left(\boldsymbol{\theta}^{h}\right)\right\}}{\partial \theta_{1}^{h}} & \ldots & \frac{\partial \mathrm{vec}\left\{O^{h}\left(\boldsymbol{\theta}^{h}\right)\right\}}{\partial \theta_{n_{h}}^{h}}
\end{array}\right]\right|_{\boldsymbol{\theta}^{h}=\boldsymbol{\theta}_{k-1}^{h}} .
$$

A simple way to calculate $\left.\frac{\partial \operatorname{vec}\left\{O^{h}\left(\boldsymbol{\theta}^{h}\right)\right\}}{\partial \theta_{j}^{h}}\right|_{\boldsymbol{\theta}^{h}=\boldsymbol{\theta}_{k-1}^{h}}$ is by numerical differentiation using the central difference theorem [23]

$$
\left.\frac{\partial \operatorname{vec}\left\{O^{h}\left(\boldsymbol{\theta}^{h}\right)\right\}}{\partial \theta_{j}^{h}}\right|_{\boldsymbol{\theta}^{h}=\boldsymbol{\theta}_{k-1}^{h}}=\frac{1}{2 \delta \theta_{j, k-1}^{h}}\left[\operatorname{vec}\left\{O^{h}\left(\boldsymbol{\theta}_{k-1}^{h}+\delta_{j} \boldsymbol{\theta}_{k-1}^{h}\right)\right\}-\operatorname{vec}\left\{O^{h}\left(\boldsymbol{\theta}_{k-1}^{h}-\delta_{j} \boldsymbol{\theta}_{k-1}^{h}\right)\right\}\right],
$$

where $\delta_{j} \boldsymbol{\theta}_{k-1}^{h} \in \mathbb{R}^{n_{h}}$ is a vector whose components are zero, except for the $j$-th component which is equal to $\delta \theta_{j, k-1}^{h}$.

\subsection{Mode-based approach}

Notice that the computation of the minimization problem can be cumbersome even though a reduced set of parameters $\boldsymbol{\theta}^{h}$ is involved, which is explained by the fact that the size of the matrix $O^{h}\left(\boldsymbol{\theta}^{h}\right)$ is usually large. To solve this issue, a model reduction technique is employed which makes use of the concept of reduced mode expansion. In this framework, the vector of nodal displacements $\mathbf{q}^{h}$ of the FE mesh of the structure is expressed in terms of mode shape vectors $\left\{\boldsymbol{\phi}_{j}^{h}\right\}_{j}$. These are solutions of the following generalized eigenproblem

$$
\mathbf{K}^{h} \boldsymbol{\phi}_{j}^{h}=\left(\omega_{j}^{h}\right)^{2} \mathbf{M}^{h} \boldsymbol{\phi}_{j}^{h}
$$

where $\left\{\omega_{j}^{h}\right\}_{j}$ are to be understood as the eigenpulsations of the vibrating structure. The reduced mode expansion hence consists in expressing the vector of nodal displacements $\mathbf{q}^{h}$ using a reduced set of mode shape vectors $\left\{\tilde{\boldsymbol{\phi}}_{j}^{h}\right\}_{j=1 \cdots n_{r}}$ which is extracted from the full set $\left\{\boldsymbol{\phi}_{j}^{h}\right\}_{j=1, \cdots, n}$, i.e., $\left\{\tilde{\boldsymbol{\phi}}_{j}^{h}\right\}_{j=1, \cdots, n_{r}} \subset\left\{\boldsymbol{\phi}_{j}^{h}\right\}_{j=1, \cdots, n}$ where $n_{r} \ll n$. The reduced mode expansion is expressed as follows

$$
\mathbf{q}^{h}(t) \approx \sum_{j=1}^{n_{r}} \tilde{\boldsymbol{\phi}}_{j}^{h} \tilde{q}_{j}^{h}(t)=\tilde{\boldsymbol{\Phi}}^{h} \tilde{\mathbf{q}}^{h}(t)
$$

where $\left\{\tilde{q}_{j}^{h}\right\}_{j}$ have the meaning of generalized coordinates; also, $\tilde{\boldsymbol{\Phi}}^{h}$ and $\tilde{\mathbf{q}}^{h}(t)$ are expressed as $\tilde{\boldsymbol{\Phi}}^{h}=\left[\tilde{\boldsymbol{\phi}}_{1}^{h} \cdots \tilde{\boldsymbol{\phi}}_{n_{r}}^{h}\right]$ and $\tilde{\mathbf{q}}^{h}(t)=\left[\tilde{q}_{1}^{h}(t) \cdots \tilde{q}_{n_{r}}^{h}(t)\right]^{T}$. As a result, a reduced extended observability matrix $\tilde{O}^{h}\left(\tilde{\boldsymbol{\theta}}^{h}\right)$, having a size of $(p+1) r \times 2 n_{r}$, can be expressed as

$$
\tilde{O}^{h}\left(\tilde{\boldsymbol{\theta}}^{h}\right)=\left[\begin{array}{c}
\tilde{\mathbf{C}}^{h} \\
\tilde{\mathbf{C}}^{h} \tilde{\mathbf{A}}^{h} \\
\vdots \\
\tilde{\mathbf{C}}^{h}\left(\tilde{\mathbf{A}}^{h}\right)^{p}
\end{array}\right]
$$

where $\tilde{\mathbf{A}}^{h}$ and $\tilde{\mathbf{C}}^{h}$ are matrices whose determination involves projecting the second-order differential equation (14) onto the reduced basis $\left\{\tilde{\boldsymbol{\phi}}_{j}^{h}\right\}_{j}$ through Galerkin procedure. This is done by left multiplying this equation by $\left(\tilde{\boldsymbol{\Phi}}^{h}\right)^{T}$ and invoking the orthogonality properties of the mode shape vectors - i.e., $\left(\tilde{\boldsymbol{\Phi}}^{h}\right)^{T} \mathbf{M}^{h} \tilde{\boldsymbol{\Phi}}^{h}=\mathbf{I}$ and $\left(\tilde{\boldsymbol{\Phi}}^{h}\right)^{T} \mathbf{K}^{h} \tilde{\boldsymbol{\Phi}}^{h}=$ $\operatorname{diag}\left\{\left(\tilde{\omega}_{j}^{h}\right)^{2}\right\}_{j}$ - as well as the fact that the matrix $\left(\tilde{\boldsymbol{\Phi}}^{h}\right)^{T} \gamma^{h} \tilde{\mathbf{\Phi}}^{h}$ is assumed to be diagonal. As a result, one has

$$
\ddot{\tilde{\mathbf{q}}}^{h}(t)+\operatorname{diag}\left\{2 \tilde{\xi}_{j}^{h} \tilde{\omega}_{j}^{h}\right\}_{j} \dot{\tilde{\mathbf{q}}}^{h}(t)+\operatorname{diag}\left\{\left(\tilde{\omega}_{j}^{h}\right)^{2}\right\}_{j} \tilde{\mathbf{q}}^{h}(t)=\tilde{\mathbf{v}}^{h}(t),
$$


where the parameters $\left\{\tilde{\xi}_{j}^{h}\right\}_{j}$ denote modal damping ratios. Expressions of the matrices $\tilde{\mathbf{A}}^{h}$ and $\tilde{\mathbf{C}}^{h}$ in the corresponding discrete-time state-space model follow as

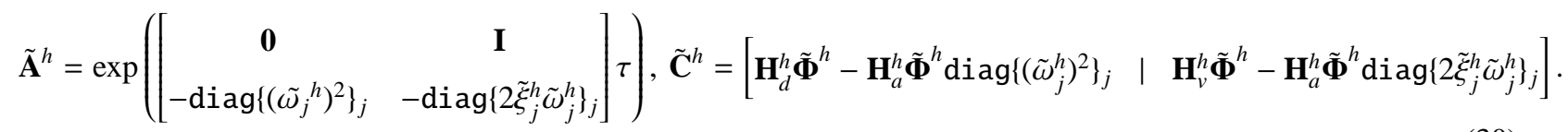

By considering Eqs. (16) and (28), the minimization problem involving the reduced extended observability matrix $\tilde{O}^{h}\left(\boldsymbol{\theta}^{h}\right)$ can be expressed as

$$
\tilde{\boldsymbol{\theta}}^{h}=\operatorname{argmin}\|\tilde{\mathbf{r}}\|_{2}^{2}
$$

where

$$
\tilde{\mathbf{r}}=\left[\mathbf{I}_{2 n_{r}} \otimes\left(\mathbf{I}_{(p+1) r}-\hat{O} \hat{O}^{\dagger}\right)\right] \operatorname{vec}\left\{\tilde{O}^{h}\left(\tilde{\boldsymbol{\theta}}^{h}\right)\right\} .
$$

Similarly as in (22), the Gauss-Newton iteration is defined as

$$
\tilde{\boldsymbol{\theta}}_{k}^{h}=\tilde{\boldsymbol{\theta}}_{k-1}^{h}-\mathcal{J}_{\tilde{\mathbf{r}}_{k}}^{\dagger} \tilde{\mathbf{r}}_{k}
$$

where the residual $\tilde{\mathbf{r}}_{k}$ writes as

$$
\tilde{\mathbf{r}}_{k}=\left[\mathbf{I}_{2 n_{r}} \otimes\left(\mathbf{I}_{(p+1) r}-\hat{O} \hat{O}^{\dagger}\right)\right] \operatorname{vec}\left\{\tilde{O}^{h}\left(\tilde{\boldsymbol{\theta}}_{k-1}^{h}\right)\right\} .
$$

It is understood that the parameters $\tilde{\boldsymbol{\theta}}^{h}$ are almost equal to those involved in the original minimization problem (20), i.e., $\tilde{\boldsymbol{\theta}}^{h} \approx \boldsymbol{\theta}^{h}$. For simplicity of notation, only $\boldsymbol{\theta}^{h}$ instead of $\tilde{\boldsymbol{\theta}}^{h}$ is used in the following, neglecting the tilde sign.

\section{Uncertainty quantification}

Analyzing the statistical uncertainty of identified parameters is mandatory to assess the quality of the estimates from the data. When estimated from a finite number of data samples, not the "true" parameters of the system are obtained, but estimates that are naturally subject to variance errors depending on the data and the estimation method, due to unknown noise inputs, measurement noise and finite data length.

A variance analysis of the system matrices obtained from stochastic subspace identification has been made in $[9,10]$ by computing a sample covariance that is closely linked to the measurements and propagating it to the system matrices through a sensitivity analysis. In this section, the variance analysis of the updated structural parameters from the FE-based SF method of the previous section is made.

The following notation is used. Let $Y$ be a matrix-valued function of $\hat{X}$. Expression of its covariance follows from a Taylor approximation

$$
\operatorname{vec}\{Y(\hat{X})\} \approx \operatorname{vec}\{Y(X)\}+\mathcal{J}_{Y, X} \operatorname{vec}\{\hat{X}-X\} \quad \Rightarrow \quad \operatorname{cov}(Y(\hat{X})) \approx \mathcal{J}_{Y, X} \hat{\Sigma}_{X} \mathcal{J}_{Y, X}^{T},
$$

where $\mathcal{J}_{Y, X}$ is the sensitivity matrix, defined by $\mathcal{J}_{Y, X}=\partial \operatorname{vec}\{Y(X)\} / \partial \operatorname{vec}\{X\}$. A consistent estimate is obtained by replacing, in the sensitivity matrix, the theoretical variables $X$ with consistent estimates $\hat{X}$ issued from data. Using 
delta notation, theoretical first-order perturbations are defined, yielding

$$
\operatorname{vec}\{\Delta Y\}=\mathcal{J}_{Y, X} \operatorname{vec}\{\Delta X\}
$$

Using this relationship, a perturbation $\Delta \hat{\mathcal{H}}$ of the Hankel matrix $\hat{\mathcal{H}}$ is propagated towards the observability matrix $\hat{O}$ and ultimately the structural parameters through the Gauss-Newton algorithm to obtain the respective sensitivity matrices. Together with a sample covariance $\hat{\Sigma}_{\mathcal{H}}$ of the Hankel matrix, which can be easily computed on the measurements, the covariance of the structural parameters is obtained through (35). Since the output-covariances of the data and the Hankel matrix estimate are asymptotically Gaussian (for $N \rightarrow \infty$ ) [24], the statistical delta method ensures that each variable in the computation chain - down to the desired structural parameters - is also asymptotically Gaussian [25], and that the respective covariance estimate in Eq. (35) is exact for $N \rightarrow \infty$.

The expressions of the covariances of the Hankel matrix and observability matrix have been derived in past works $[8,9]$ and are recalled here for the sake of clarity.

\subsection{Covariance of the Hankel matrix}

The derivation of an estimate of the covariance of the Hankel matrix, namely $\hat{\Sigma}_{\mathcal{H}}$, is achieved by splitting the data matrices $\mathcal{Y}^{+}$and $\mathcal{Y}^{-}$in (11) into $n_{b}$ blocks, as follows

$$
\boldsymbol{y}^{+}=\left[\begin{array}{llll}
y_{1}^{+} & y_{2}^{+} & \ldots & y_{n_{b}}^{+}
\end{array}\right], \quad \boldsymbol{y}^{-}=\left[\begin{array}{llll}
y_{1}^{-} & \boldsymbol{y}_{2}^{-} & \ldots & \boldsymbol{y}_{n_{b}}^{-}
\end{array}\right]
$$

For each pair of sub-matrices $\left(\mathcal{Y}_{j}^{+}, \mathcal{Y}_{j}^{-}\right)$, the corresponding Hankel matrix $\hat{\mathcal{H}}_{j}$ can be estimated as in Eq. (10) as

$$
\hat{\mathcal{H}}_{j}=\frac{1}{N_{b}} \mathcal{Y}_{j}^{+}\left(\mathcal{Y}_{j}^{-}\right)^{T},
$$

where $N_{b}=N / n_{b}$. As a result, $\hat{\mathcal{H}}=\left(1 / n_{b}\right) \sum_{j=1}^{n_{b}} \hat{\mathcal{H}}_{j}$ and the estimate $\hat{\Sigma}_{\mathcal{H}}$ is given by

$$
\hat{\Sigma}_{\mathcal{H}}=\frac{1}{n_{b}\left(n_{b}-1\right)} \sum_{j=1}^{n_{b}}\left(\operatorname{vec}\left\{\hat{\mathcal{H}}_{j}\right\}-\operatorname{vec}\{\hat{\mathcal{H}}\}\right)\left(\operatorname{vec}\left\{\hat{\mathcal{H}}_{j}\right\}-\operatorname{vec}\{\hat{\mathcal{H}}\}\right)^{T}
$$

\subsection{Covariance of the observability matrix}

A perturbation $\Delta \mathcal{H}$ of the Hankel matrix is propagated towards the observability matrix. In order to obtain $\Delta \hat{O}$ from an estimate of $O$ (see Eq. (13)), the sensitivities of the left singular vectors $\mathbf{U}_{1}$ (see Eq. (13)) are to be derived, as follows [10]

$$
\operatorname{vec}\left\{\Delta \mathbf{U}_{1}\right\}=\mathcal{J}_{\mathbf{U}_{1}, \mathcal{H}} \operatorname{vec}\{\Delta \hat{\mathcal{H}}\}=\left[\begin{array}{c}
\mathcal{B}_{1} C_{1} \\
\vdots \\
\mathcal{B}_{2 n} C_{2 n}
\end{array}\right] \operatorname{vec}\{\Delta \hat{\mathcal{H}}\}
$$

where

$$
\mathcal{B}_{i}=\left[\mathbf{I}_{(p+1) r}+\frac{\hat{\mathcal{H}}}{\sigma_{i}} \mathcal{D}_{i}\left(\frac{\hat{\mathcal{H}}^{T}}{\sigma_{i}}-\left[\begin{array}{c}
\mathbf{0}_{q r-1,(p+1) r} \\
\mathbf{u}_{i}^{T}
\end{array}\right]\right) \mid \frac{\hat{\mathcal{H}}}{\sigma_{i}} \mathcal{D}_{i}\right], \quad \mathcal{D}_{i}=\left(\mathbf{I}_{q r}+\left[\begin{array}{c}
\mathbf{0}_{q r-1,(p+1) r} \\
2 \mathbf{v}_{i}^{T}
\end{array}\right]-\frac{\hat{\mathcal{H}}^{T} \hat{\mathcal{H}}}{\sigma_{i}^{2}}\right)^{-1}
$$


and

$$
C_{i}=\frac{1}{\sigma_{i}}\left[\begin{array}{c}
\left(\mathbf{I}_{(p+1) r}-\mathbf{u}_{i} \mathbf{u}_{i}^{T}\right)\left(\mathbf{v}_{i}^{T} \otimes \mathbf{I}_{(p+1) r}\right) \\
\left(\mathbf{I}_{q r}-\mathbf{v}_{i} \mathbf{v}_{i}^{T}\right)\left(\mathbf{I}_{q r} \otimes \mathbf{u}_{i}^{T}\right)
\end{array}\right],
$$

where $\mathbf{u}_{i}$ and $\mathbf{v}_{i}$ stand for the $i$-th left and right singular vectors, respectively. Then, an estimate of the covariance matrix of $\hat{O}$ can be derived from (35) as

$$
\hat{\Sigma}_{O}=\mathcal{J}_{\mathbf{U}_{1}, \mathcal{H}} \hat{\Sigma}_{\mathcal{H}} \mathcal{J}_{\mathbf{U}_{1}, \mathcal{H}}^{T}
$$

\subsection{Covariance matrix of the structural parameters}

The purpose of the present section is to link the parameter perturbation $\Delta \boldsymbol{\theta}_{k}^{h}$ with the perturbation of the observability matrix. The main result is that

$$
\Delta \boldsymbol{\theta}_{k}^{h}=\mathcal{M}_{k} \operatorname{vec}\{\Delta \hat{O}\}
$$

where $\mathcal{M}_{k} \in \mathbb{R}^{n_{h} \times 2 n(p+1) r}$. It is defined iteratively by

$$
\mathcal{M}_{k}=\mathcal{M}_{k-1}-\left(\tilde{\mathbf{r}}_{k}^{T} \otimes \mathbf{I}_{h_{h}}\right) \mathcal{L}_{k} \mathcal{N}_{k}-\mathcal{J}_{\tilde{\mathbf{r}}_{k}}^{\dagger} Q_{k}
$$

where $\mathcal{M}_{0}=\mathbf{0}_{n_{h}, 2 n(p+1) r}$, while matrices $\mathcal{L}_{k}, \mathcal{N}_{k}$ and $Q_{k}$ will be detailed in the following. Hence, an estimate of the covariance matrix of $\boldsymbol{\theta}_{k}^{h}$ is given by

$$
\hat{\Sigma}_{\theta_{k}^{h}}=\mathcal{M}_{k} \hat{\Sigma}_{O} \mathcal{M}_{k}^{T},
$$

where $\hat{\Sigma}_{\boldsymbol{\theta}_{k}^{l}}$ is the parameter covariance matrix for the $k$-th iteration.

In a constructive proof, relation (44) is derived by induction. Obviously, (44) holds for $k=0$, since $\Delta \boldsymbol{\theta}_{0}^{h}=\mathbf{0}_{n_{h}}$ due to the deterministic nature of the initial value. Then, for a fixed $k$ with $k \geq 1$, assume that (44) holds for $k-1$, i.e.

$$
\Delta \boldsymbol{\theta}_{k-1}^{h}=\mathcal{M}_{k-1} \operatorname{vec}\{\Delta \hat{O}\}
$$

and the relation (44) for $k$ is proved as follows.

The starting point is to consider a perturbation on the iteration $\boldsymbol{\theta}_{k}^{h}=\boldsymbol{\theta}_{k-1}^{h}-\mathcal{J}_{\tilde{\mathbf{r}}_{k}}^{\dagger} \tilde{\mathbf{r}}_{k}$ in (33). Separating zeroth- and first-order terms and using the fact that $\operatorname{vec}\{\mathbf{A B}\}=\left(\mathbf{B}^{T} \otimes \mathbf{I}_{a}\right) \operatorname{vec}\{\mathbf{A}\}$ for any matrices $\mathbf{A} \in \mathbb{R}^{a \times b}$ and $\mathbf{B} \in \mathbb{R}^{b \times c}$ [26], this yields

$$
\Delta \boldsymbol{\theta}_{k}^{h}=\Delta \boldsymbol{\theta}_{k-1}^{h}-\left(\tilde{\mathbf{r}}_{k}^{T} \otimes \mathbf{I}_{h_{h}}\right) \operatorname{vec}\left\{\Delta \mathcal{J}_{\tilde{\mathbf{r}}_{k}}^{\dagger}\right\}-\mathcal{J}_{\tilde{\mathbf{r}}_{k}}^{\dagger} \Delta \tilde{\mathbf{r}}_{k} .
$$

From Appendix B, both vectors vec $\left\{\Delta \mathcal{J}_{\tilde{\mathbf{r}}_{k}}^{\dagger}\right\}$ and $\Delta \tilde{\mathbf{r}}_{k}$ can be written in terms of $\operatorname{vec}\{\Delta \hat{O}\}$ as

$$
\begin{aligned}
\operatorname{vec}\left\{\Delta \mathcal{J}_{\tilde{\mathbf{r}}_{k}}^{\dagger}\right\} & =\mathcal{L}_{k} \mathcal{N}_{k} \operatorname{vec}\{\Delta \hat{O}\}, \\
\Delta \tilde{\mathbf{r}}_{k} & =Q_{k} \operatorname{vec}\{\Delta \hat{O}\},
\end{aligned}
$$

where 
- Following the calculations in Appendix B with $\mathbf{X}=\tilde{O}^{h}\left(\boldsymbol{\theta}_{k-1}^{h}\right)$ and $\mathbf{Y}=\tilde{\mathbf{r}}_{k}, Q_{k}$ is expressed by

$$
Q_{k}=-\left[\left(\hat{O}^{\dagger} \tilde{O}^{h}\left(\boldsymbol{\theta}_{k-1}^{h}\right)\right)^{T} \otimes \mathbf{I}_{(p+1) r}\right]-\left[\left(\tilde{O}^{h}\left(\boldsymbol{\theta}_{k-1}^{h}\right)\right)^{T} \otimes \hat{O}\right] \mathcal{K}+\left[\mathbf{I}_{2 n_{r}} \otimes\left(\mathbf{I}_{(p+1) r}-\hat{O} \hat{O}^{\dagger}\right)\right] \mathcal{J}_{\tilde{O}^{h}, \boldsymbol{\theta}_{k-1}^{h}} \mathcal{M}_{k-1}
$$

where $\mathcal{J}_{\tilde{O}^{h}, \theta_{k-1}^{h}}$ is defined as in (24) and matrix $\mathcal{K}$ satisfies $\operatorname{vec}\left\{\Delta \hat{O}^{\dagger}\right\}=\mathcal{K} \operatorname{vec}\{\Delta \hat{O}\}$ with

$$
\mathcal{K}=\left\{-\left[\left(\hat{O} \hat{O}^{\dagger}\right)^{T} \otimes\left(\hat{O}^{T} \hat{O}\right)^{-1}\right]+\left[\mathbf{I}_{(p+1) r} \otimes\left(\hat{O}^{T} \hat{O}\right)^{-1}\right]\right\} \mathcal{P}_{(p+1) r, 2 n}-\left[\left(\hat{O}^{\dagger}\right)^{T} \otimes \hat{O}^{\dagger}\right]
$$

following from Appendix A with permutation matrix $\mathcal{P}_{(p+1) r, 2 n}$ defined in (A.3).

- Matrix $\mathcal{L}_{k}$ satisfies $\operatorname{vec}\left\{\Delta \mathcal{J}_{\tilde{r}_{k}}^{\dagger}\right\}=\mathcal{L}_{k} \operatorname{vec}\left\{\Delta \mathcal{J}_{\tilde{\mathbf{r}}_{k}}\right\}$ with

$$
\mathcal{L}_{k}=\left\{-\left[\left(\mathcal{J}_{\tilde{\mathbf{r}}_{k}} \mathcal{J}_{\tilde{\mathbf{r}}_{k}}^{\dagger}\right)^{T} \otimes\left(\mathcal{J}_{\tilde{\mathbf{r}}_{k}}^{T} \mathcal{J}_{\tilde{\mathbf{r}}_{k}}\right)^{-1}\right]+\left[\mathbf{I}_{2(p+1) r n_{r}} \otimes\left(\mathcal{J}_{\tilde{\mathbf{r}}_{k}}^{T} \mathcal{J}_{\tilde{\mathbf{r}}_{k}}\right)^{-1}\right]\right\} \mathcal{P}_{2(p+1) r n_{r}, n_{h}}-\left[\left(\mathcal{J}_{\tilde{\mathbf{r}}_{k}}^{\dagger}\right)^{T} \otimes \mathcal{J}_{\tilde{\mathbf{r}}_{k}}^{\dagger}\right]
$$

following from Appendix A.

- Matrix $\mathcal{N}_{k}$ satisfies $\operatorname{vec}\left\{\Delta \mathcal{J}_{\tilde{\mathbf{r}}_{k}}\right\}=\mathcal{N}_{k} \operatorname{vec}\{\Delta \hat{O}\}$ with

$$
\mathcal{N}_{k}=\left[\begin{array}{c}
\mathcal{N}_{1, k} \\
\mathcal{N}_{2, k} \\
\vdots \\
\mathcal{N}_{n_{h}, k}
\end{array}\right],
$$

where $\mathcal{N}_{j, k}$ is derived from Appendix B with $\mathbf{X}=\mathcal{J}_{\tilde{O}^{h}, \theta_{j, k-1}^{h}}^{*}$, where $\mathcal{J}_{\tilde{O}^{h}, \theta_{j, k-1}^{h}}^{*} \in \mathbb{R}^{(p+1) r \times 2 n_{r}}$ is such that $\operatorname{vec}\left\{\mathcal{J}_{\tilde{O}^{h}, \theta_{j, k-1}^{h}}^{*}\right\}=$ $\mathcal{J}_{\tilde{O}^{h}, \theta_{j, k-1}^{h}}$, and $\mathbf{Y}=\left[\mathbf{I}_{2 n_{r}} \otimes\left(\mathbf{I}_{(p+1) r}-\hat{O} \hat{O}^{\dagger}\right)\right] \mathcal{J}_{\tilde{O}^{h}, \theta_{j k-1}^{h}}$ being the $j$-th column of $\mathcal{J}_{\tilde{\mathbf{r}}_{k}}$ as in (23). It follows

$$
\mathcal{N}_{j, k}=-\left[\left(\hat{O}^{\dagger} \mathcal{J}_{\tilde{O}^{h}, \theta_{j, k-1}^{h}}^{*}\right)^{T} \otimes \mathbf{I}_{(p+1) r}\right]-\left[\left(\mathcal{J}_{\tilde{O}^{h}, \theta_{j, k-1}^{h}}^{*}\right)^{T} \otimes \hat{O}\right] \mathcal{K}+\left[\mathbf{I}_{2 n_{r}} \otimes\left(\mathbf{I}_{(p+1) r}-\hat{O} \hat{O}^{\dagger}\right)\right] \mathcal{J}_{\mathcal{J}_{\tilde{O}^{h}, \theta_{j, k-1}^{h}}, \boldsymbol{\theta}_{k-1}^{h}} \mathcal{M}_{k-1}
$$

Matrix $\mathcal{J}_{\mathcal{J}_{\tilde{O}^{h}, \theta_{j, k-1}^{h}}^{*} \theta_{k-1}^{h}}$ satisfies vec $\left\{\Delta \mathcal{J}_{\tilde{O}^{h}, \theta_{j, k-1}^{h}}^{*}\right\}=\mathcal{J}_{\mathcal{J}_{h^{h}, j_{j, k-1}^{h}}^{*}} \theta_{k-1}^{h} \operatorname{vec}\left\{\Delta \theta_{k-1}^{h}\right\}$ and is obtained by numerical differentiation similar to (25).

Finally, introducing Eqs. (47), (49) and (50) into Eq. (48) proves the assertion for the perturbation on the parameter at iteration $k$ in (44) with $\mathcal{M}_{k}$ defined as in Eq. (45). The estimate of the covariance matrix of the structural parameters $\hat{\Sigma}_{\theta_{k}^{l}}$ (see Eq. (46)) can thus be obtained for the $k$-th iteration of the Gauss-Newton algorithm.

\section{Numerical validation}

\subsection{Introduction}

The proposed approach is used to estimate the variances of some mechanical parameters of a vibrating cantilever beam whose measurement data result from a fine FE mesh with known characteristics, see Figure 2. The characteristics of the beam are: length of $1 \mathrm{~m}$, cross-section of $0.0249 \mathrm{~m} \times 0.0053 \mathrm{~m}$, Young's modulus of 200GPa, density of 
$7,850 \mathrm{~kg} / \mathrm{m}^{3}$ and Poisson's ratio of 0.3 . The damping matrix is defined so that $\boldsymbol{\gamma}=\mathbf{M}+10^{-6} \mathbf{K}$, where $\mathbf{M}$ and $\mathbf{K}$ are the mass and stiffness matrices of the beam. The reference FE mesh is composed of $3 \mathrm{D}$ tetrahedral elements with three DOFs per node and contains 5,505 DOFs, see Figure 2. The eigenfrequencies of the 3D FE beam are reported in Table 1. The output data (vector $\mathbf{y}_{k}$ ) refer to the transverse displacement of the structure which is recorded by means

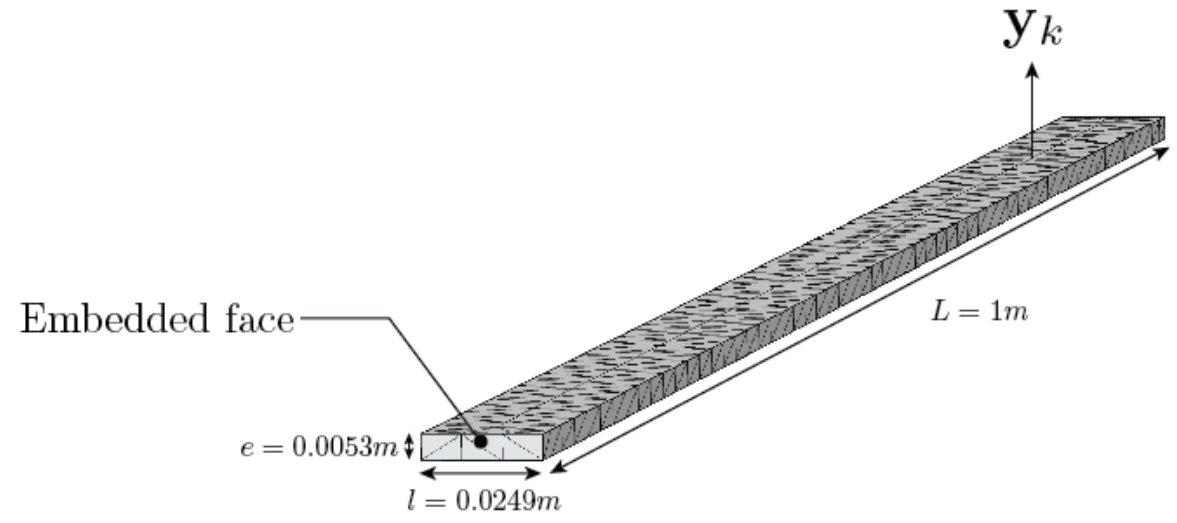

Figure 2: 3D FE model of the beam.

of one displacement sensor located at $1 \mathrm{~m}$ from the clamped end. The sampling frequency used for measuring these data is $1,280 \mathrm{~Hz}$ and the number of samples is $N=12,800$. A Gaussian random noise is added to the output with a signal to noise ratio of $20 \mathrm{~dB}$.

\begin{tabular}{|c|c|c|c|c|c|}
\hline \multirow[b]{3}{*}{ Mode } & \multirow{3}{*}{$\begin{array}{l}\text { 3D beam } \\
f[\mathrm{~Hz}]\end{array}$} & \multicolumn{4}{|c|}{ 1D beam } \\
\hline & & \multicolumn{2}{|c|}{$E=160 G P a$} & \multicolumn{2}{|c|}{$E=200.21 G P a$} \\
\hline & & $f[H z]$ & Err [\%] & $f[H z]$ & Err $[\%]$ \\
\hline 1 & 4.3293 & 3.8653 & 10.72 & 4.3238 & 0.13 \\
\hline 2 & 27.1280 & 24.2241 & 10.70 & 27.0975 & 0.11 \\
\hline 3 & 75.9513 & 67.8433 & 10.66 & 75.8907 & 0.08 \\
\hline 4 & 148.8102 & 133.0385 & 10.60 & 148.8193 & 0.01 \\
\hline 5 & 245.9611 & 220.2667 & 10.45 & 246.3943 & 0.18 \\
\hline 6 & 367.3624 & 329.9845 & 10.17 & 369.1267 & 0.48 \\
\hline 7 & 512.9839 & 462.9843 & 9.75 & 517.9027 & 0.96 \\
\hline
\end{tabular}

Table 1: Comparison between the eigenfrequencies of the 3D FE beam and those of the updated 1D model.

\subsection{Young's modulus updating and standard deviation estimation}

The FE-based SF approach and uncertainties quantification are investigated. In this framework, a coarse FE mesh based on the Euler-Bernoulli beam theory is considered as shown in Figure 3. This mesh is made up of 10 Euler- 
Bernoulli beam elements of same length with six DOFs per node. As a result, this coarse FE mesh contains $n=60$ DOFs, which appears to be small compared to the number of DOFs used to discretize the 3D structure. The number of DOFs involved can be further reduced by considering the model reduction strategy depicted in Section 2.3. In this framework, a reduced number of modes are used to describe the dynamic behavior of the $1 \mathrm{D}$ beam. The manner by which these modes are selected follows from the procedure explained in [27], which yields $n_{r}=7$ modes.

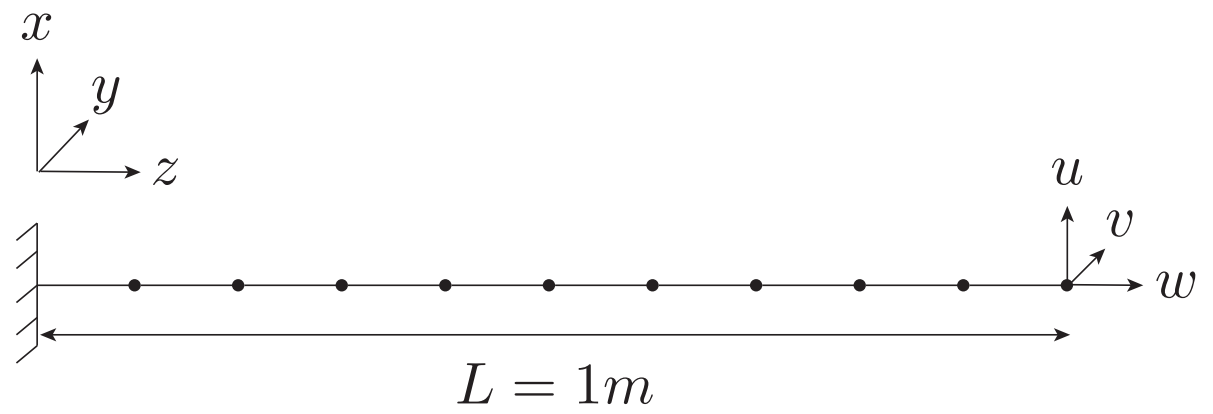

Figure 3: Coarse 1D FE mesh of the beam

The issue here consists in updating the Young's modulus - namely, $E$ - of the coarse 1D FE model, which is assumed to be unknown. This yields a single-parameter SF minimization problem, as follows

$$
E=\operatorname{argmin}\|\tilde{\mathbf{r}}\|_{2}^{2} \quad \text { where } \quad \tilde{\mathbf{r}}=\left[\mathbf{I}_{2 n_{r}} \otimes\left(\mathbf{I}_{(p+1) r}-\hat{O} \hat{O}^{\dagger}\right)\right] \operatorname{vec}\left\{\tilde{O}^{h}(E)\right\}
$$

where $O^{h}$ is the observability matrix issued from the 1D FE model of the beam (Figure 3). To solve Eq. (56), the algorithm procedure depicted in Section 2 can be easily implemented using MATLAB ${ }^{\circledR}$ where $p=50$ and $2 n_{r}=14$.

In order to validate the proposed method, a comparison is made with Monte-Carlo simulations [28], where a large number $n_{e}$ of output-only datasets are generated from random excitation. For each dataset, the parameter $E^{(i)}(i=$ $\left.1, \ldots, n_{e}\right)$, is computed with the SF approach, see Section 2. From the set of identified parameters, the sample standard deviation can be directly evaluated. Monte-Carlo simulation is the most accurate method but is computationally expensive, i.e., it can be impracticable for experimental applications. Hence, a set of $n_{e}=1,000$ output signals are obtained by applying random state and input noises to the 3D FE beam. The minimization problem (56) is solved for each set of output signals, leading to 1,000 values of the Young's modulus.

The sample mean of the updated values of the Young's modulus in the Monte-Carlo simulations - namely $\bar{E}=$ $1 / n_{e} \sum_{i=1}^{n_{e}} E^{(i)}$-, which results from an initial value $E=160 G P a$, is found to be $\bar{E}=200.21 G P a$ after 20 iterations as shown in Figure 4. The eigenfrequencies of the 1D beam, which result from the initial and updated Young's modulus, are reported in Table 1 and compared with those of the reference 3D model.

From the uncertainty quantification method described in Section 3, the standard deviation of Young's modulus for each dataset can be estimated by

$$
\sigma_{\Delta, S F}^{E^{(i)}}=\sqrt{\hat{\Sigma}_{E^{(i)}}}
$$




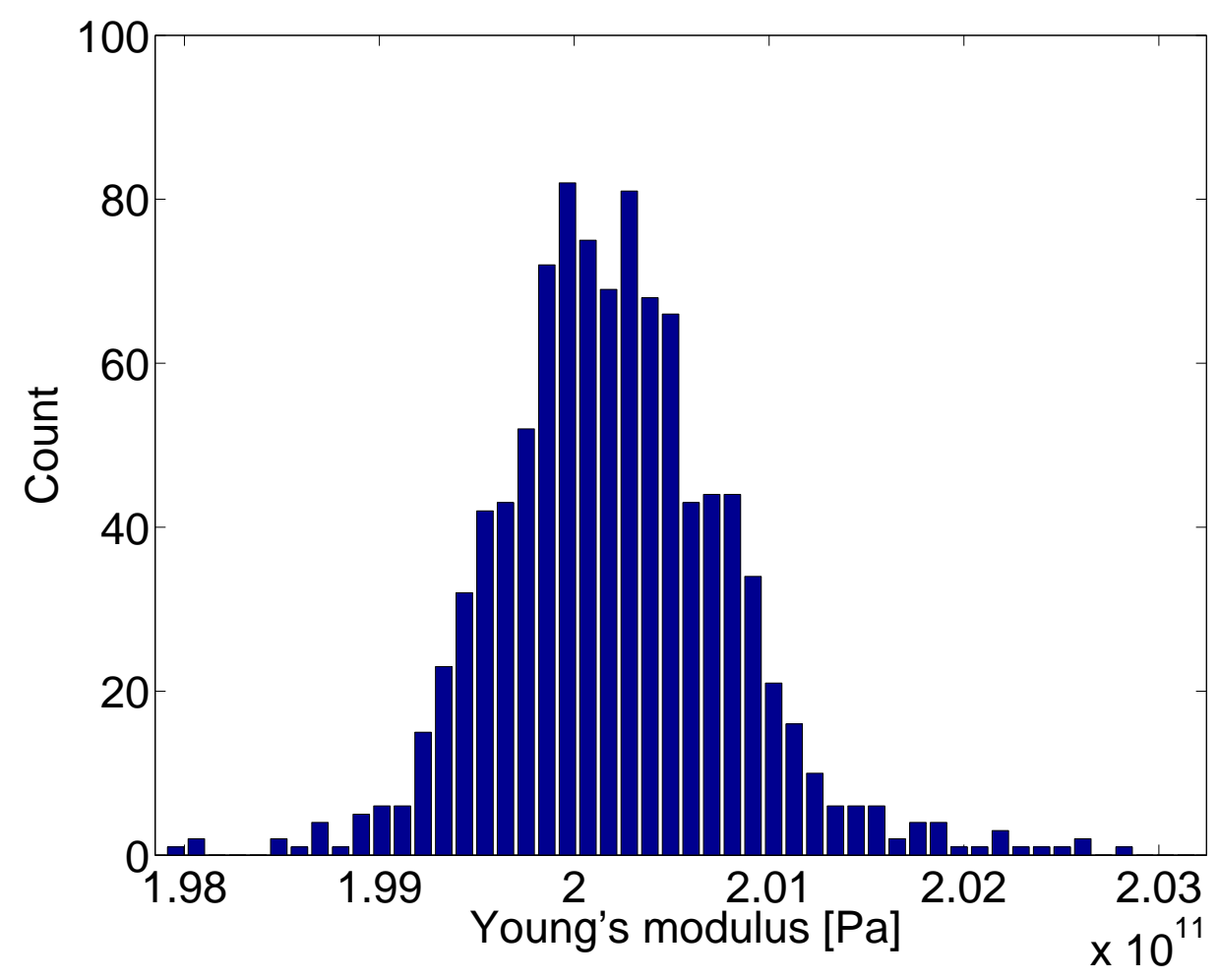

Figure 4: Young's modulus distribution obtained from Monte-Carlo simulations.

where $\hat{\Sigma}_{E^{(i)}}$ is the estimate of the variance of the Young's modulus estimated from dataset $i$, see Eq. (46). It is evaluated by using the procedure explained in Section 3, where $n_{b}=100$, and its mean is found to be $\overline{\sigma_{\Delta, S F}^{E}}=0.53 G P a$ at the last iteration of the Gauss-Newton procedure. This value should be close to the sample standard deviation of the Young's modulus obtained from the Monte-Carlo simulations, which is given by

$$
\sigma_{M C, S F}^{E}=\sqrt{\frac{1}{n_{e}-1} \sum_{i=1}^{n_{e}}\left(E^{(i)}-\bar{E}\right)^{2}},
$$

and is found to be $\sigma_{M C, S F}^{E}=0.61 G P a$. Indeed, this value is close to the estimated standard deviation with the proposed method. The difference may be explained by the approximation of the covariance estimation in (35), which is getting more precise when the number of samples $N$ in the datasets increases. In fact, $N=12,800$ for a sampling frequency of $1,280 \mathrm{~Hz}$ may be relatively low for convergence.

The distribution of the estimated standard deviations of the Young's modulus in (57) is displayed in Figure 5. The sample standard deviation of the estimated standard deviations can be computed from the Monte-Carlo simulations and is found to be $\sigma_{\sigma_{\triangle, S F}^{E}}=0.13 \mathrm{GPa}$. This represents about $25 \%$ of the estimated standard deviation. In other words, the estimation error on the standard deviation from a single dataset is about $25 \%$, which is a reasonable value for a second-order statistic. 


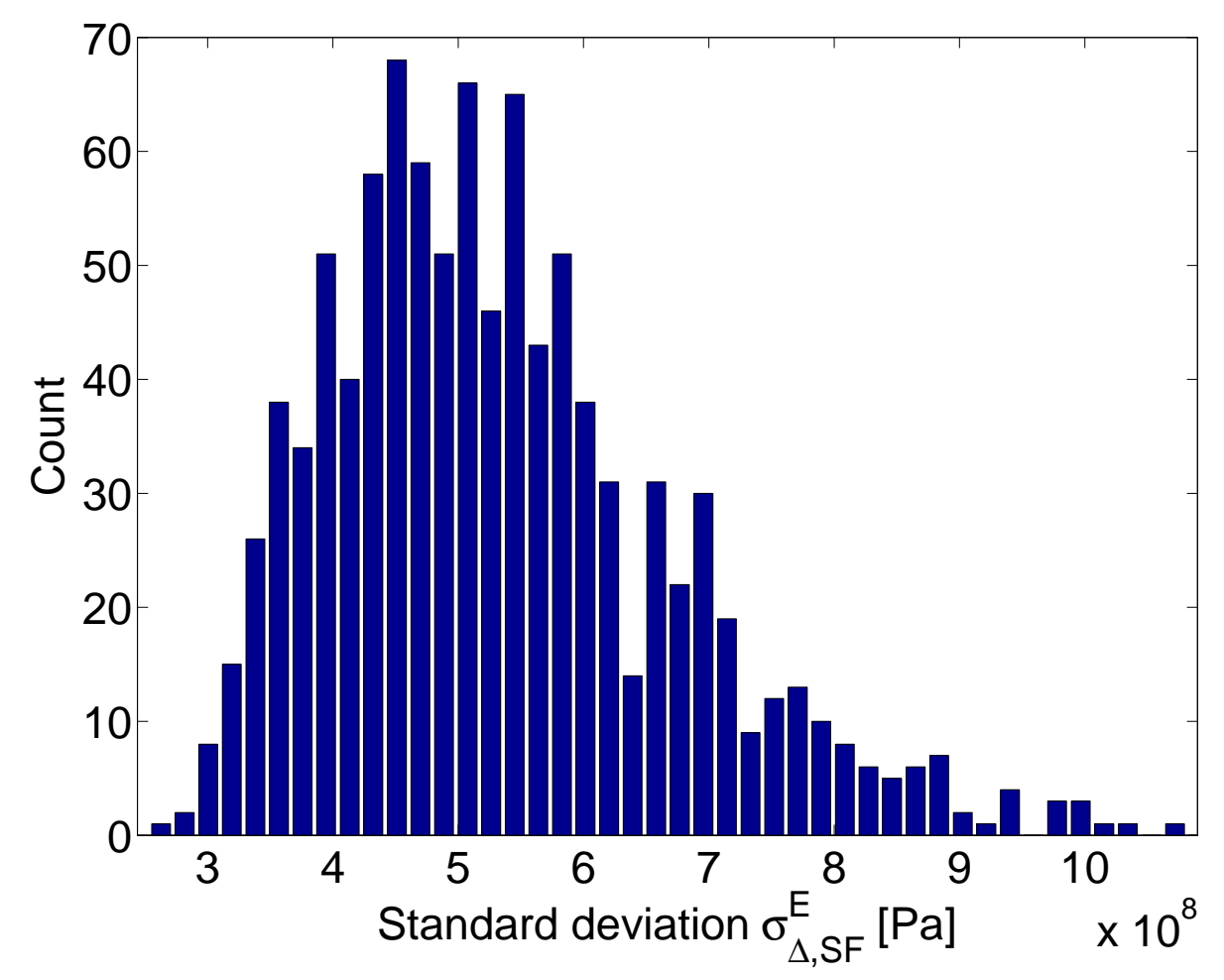

Figure 5: Distribution of the variance of the Young's modulus.

Furthermore, considering that $E$ is the only unknown parameter of the $\mathrm{FE}$ model, the standard deviations of the eigenfrequencies - namely, $f_{j}$ - can be obtained from the estimate of the variance of the Young's modulus as (see Eq. (36))

$$
\hat{\Sigma}_{f_{j}}=\mathcal{J}_{f_{j}, E} \hat{\Sigma}_{E} \mathcal{J}_{f_{j}, E}^{T},
$$

where

$$
\mathcal{J}_{f_{j}, E}=\frac{\partial f_{j}}{\partial E} .
$$

Their means are displayed in Table 2. In comparison, the sample standard deviations of the eigenfrequencies can be obtained from the Monte-Carlo simulations as

$$
\sigma_{M C, S F}^{f_{j}}=\sqrt{\frac{1}{n_{e}-1} \sum_{i=1}^{n_{e}}\left(f_{j}^{(i)}-\overline{f_{j}}\right)^{2}}, \quad \overline{f_{j}}=\frac{1}{n_{e}} \sum_{j=1}^{n_{e}} f_{j}^{(i)} .
$$

They are reported in Table 2. It can be seen that the uncertainty bounds obtained from the proposed approach are close to those provided by Monte-Carlo simulations, as expected.

Notice that the mean of the CPU time involved to solve the minimization problem (56) and to calculate the estimate of the standard deviation of the Young's modulus is $16.25 \mathrm{~s}$, which appears to be quite reasonable. Within the Monte 
Carlo framework, the CPU time involved to solve the 1,000 minimization problems is $16,942 s$, which is significantly higher than that of the proposed approach.

\begin{tabular}{cccc}
\hline mode & frequency $[\mathrm{Hz}]$ & $\sigma_{\Delta, S F}^{f^{(i)}}$ & $\sigma_{M C, S F}^{f^{(i)}}$ \\
\hline 1 & 4.3238 & 0.0058 & 0.0066 \\
2 & 27.0975 & 0.0361 & 0.0416 \\
3 & 75.8907 & 0.1011 & 0.1165 \\
4 & 148.8193 & 0.1983 & 0.2285 \\
5 & 246.3943 & 0.3283 & 0.3783 \\
6 & 369.1267 & 0.4918 & 0.5668 \\
7 & 517.9027 & 0.6900 & 0.7952 \\
\hline
\end{tabular}

Table 2: Standard deviation of the eigenfrequencies.
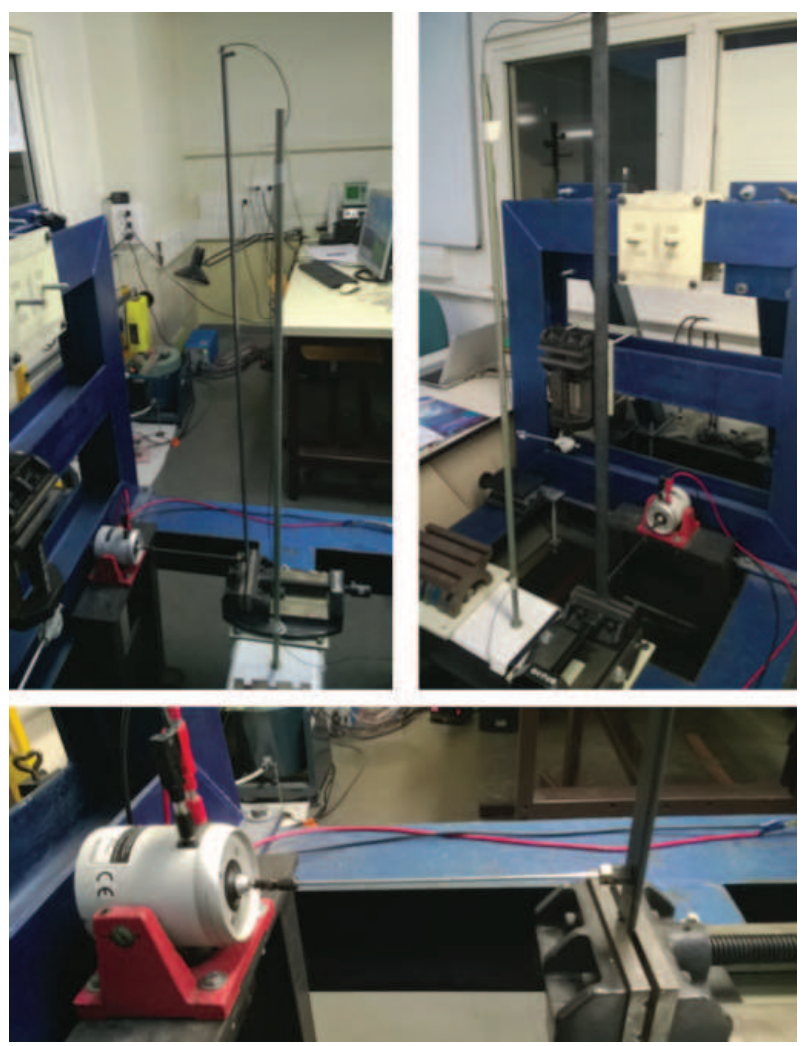

Figure 6: Experimental set-up of the beam. 


\section{Experimental application}

An application on a real experimental beam is carried out to investigate further the accuracy of the uncertainty estimation. The experimental structure is shown in Figure 6, with dimensions which are similar to those depicted in Section 4. The density, Poisson's ratio and damping of the structure are supposed to be known. The excitation is a random noise which is generated by a shaker located at $3.5 \mathrm{~cm}$ from the clamped end. The output data are recorded through a mono-axial accelerometer located on the free end of the beam.

Output data are sampled at a rate of $32,768 \mathrm{~Hz}$. The number of samples is set to be $N=496,000$, which results in a measurement time of approximately $15 \mathrm{~s}$. Afterwards, the data are filtered with a low-pass filter with a cut-off frequency of $300 \mathrm{~Hz}$ and resampled at $512 \mathrm{~Hz}$, which reduced the number of samples to $N=7750$. The pre-processed data are used to update the Young's modulus of the 1D FE beam (see Figure 3), which is unknown. The parameters used in the updating procedure are $p=20, n=8$ and $n_{b}=100$.

As a whole, 70 runs are conducted in order to estimate 70 values of the Young's modulus by means of the proposed approach. The mean of the updated values of the Young's modulus, which results from an initial guess $E=160 \mathrm{GPa}$, is found to be $\bar{E}=188.50 \mathrm{GPa}$ after 30 iterations as shown in Figure 7. The mean of the standard deviation is found to be $\bar{\sigma}_{\Delta, S F}^{E}=0.13 G P a$.

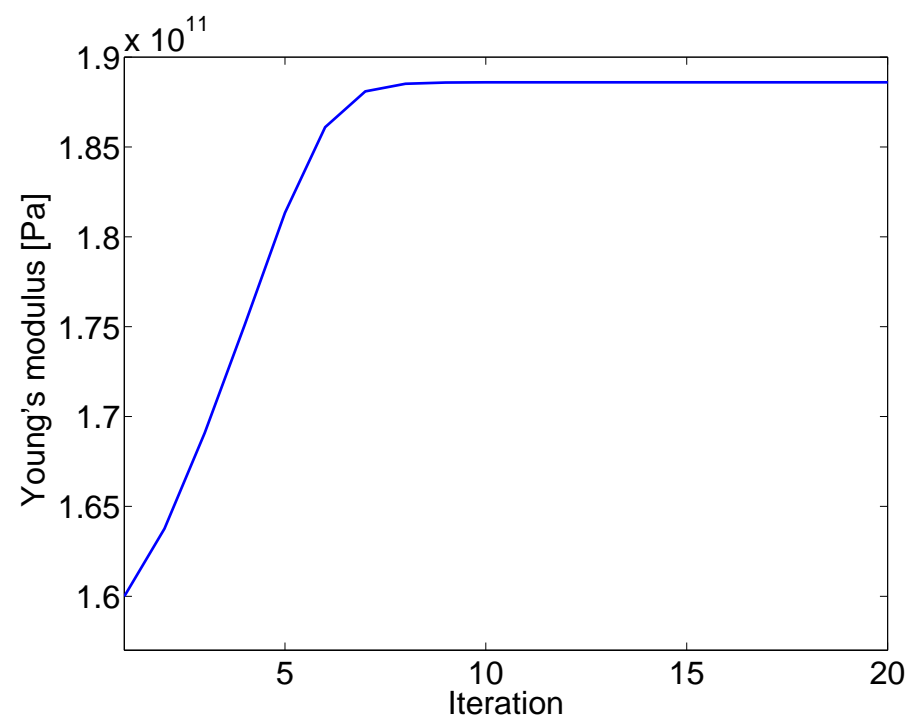

Figure 7: Overview of the iterative updating procedure, regarding the Young's modulus of the experimental beam.

Again, the Monte-Carlo procedure is carried out in order to validate the proposed approach. The sample standard deviation of the Young's modulus is computed from the 70 estimations and it is found to be $\sigma_{M C, S F}^{E}=0.15 G P a$, see Figure 8. As expected, this value appears to be closely linked with the standard deviation issued from the proposed approach. 


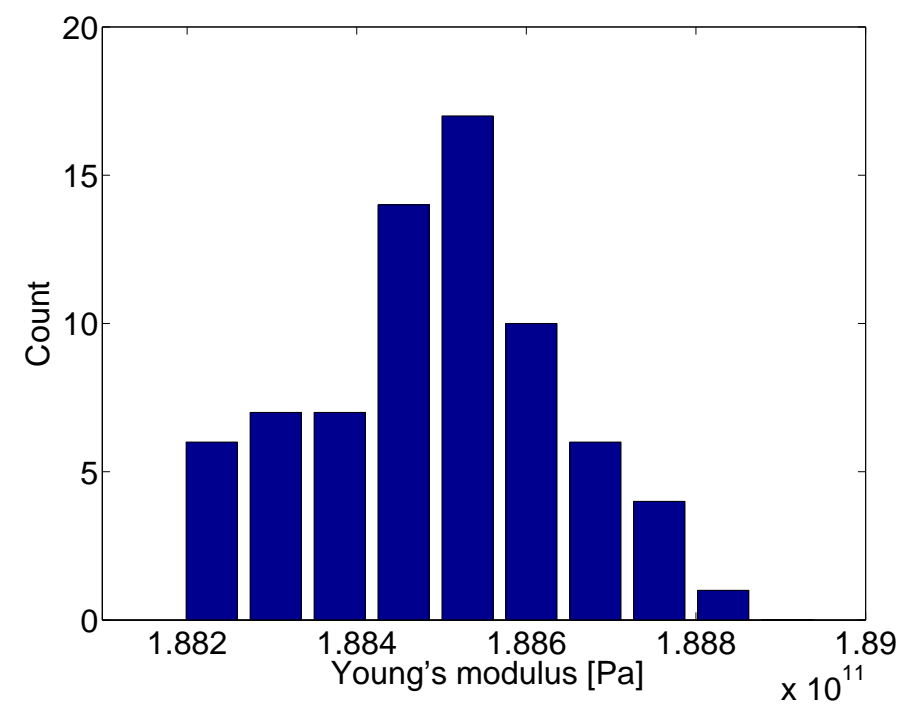

Figure 8: Young's modulus distribution obtained from Monte-Carlo simulations.

\section{Concluding remarks}

In this paper, a theoretical framework has been proposed to quantify the variance in a SF model updating procedure. In this framework, a strategy has been proposed to calculate covariance estimates when updating the parameters of a FE model from measurement data. The proposed method has been successfully validated through Monte Carlo experiments conducted on numerical and experimental beams. It has been shown that the variance estimates resulting from the proposed method are in good agreement with those resulting from sample statistics of the Monte Carlo procedure.

Variance estimation procedures have already been developed for subspace methods. Such works only addressed the simpler case of global system identification algorithms. In the current paper, first variance results were obtained for the case of iterative optimization algorithms. It is a promising approach that could be applied to other algorithms based on Gauss-Newton optimizations such as maximum likelihood approaches.

Every uncertainty quantification scheme aims at obtaining uncertainty information based on prior information and collected measurements. In a Bayesian model updating scheme as e.g. outlined in [12], the prior information, i.e. the non-calibrated FE model parameters, are considered to be random variables with a given probability distribution, and the final uncertainty is derived in a likelihood framework based on the Bayes formula in a direct computation. In the proposed approach, only the uncertainty related to the measurement data is considered, which does not require prior information about the FE parameters as in the Bayesian framework. The estimated parameters issued from SSI methods follow an asymptotically Gaussian distribution [29], leading to an asymptotically Gaussian distribution of the updated FE model parameters. Then, a prior Gaussian distribution of the initial FE model parameters could easily be incorporated in the proposed approach. 
The impact of uncertainties still constitutes an open and tough challenge in FE model updating. Future works may involve incorporating the parameter covariance matrix, at each iteration of the FE-based SF identification algorithm, in order to regularize the procedure.

\section{Appendix A. Vectorization of Moore-Penrose pseudoinverse matrix perturbation}

Consider a matrix $\mathbf{X} \in \mathbb{R}^{a \times b}$ which is assumed to be full column rank. Then the Moore-Penrose pseudoinverse $\mathbf{X}^{\dagger}$ of $\mathbf{X}$ is expressed as

$$
\mathbf{X}^{\dagger}=\left(\mathbf{X}^{T} \mathbf{X}\right)^{-1} \mathbf{X}^{T}
$$

The first-order perturbation of $\mathbf{X}^{\dagger}$ writes

$$
\begin{aligned}
\Delta \mathbf{X}^{\dagger} & =\Delta\left[\left(\mathbf{X}^{T} \mathbf{X}\right)^{-1}\right] \mathbf{X}^{T}+\left(\mathbf{X}^{T} \mathbf{X}\right)^{-1} \Delta \mathbf{X}^{T} \\
& =-\left(\mathbf{X}^{T} \mathbf{X}\right)^{-1} \Delta \mathbf{X}^{T} \mathbf{X} \mathbf{X}^{\dagger}-\mathbf{X}^{\dagger} \Delta \mathbf{X} \mathbf{X}^{\dagger}+\left(\mathbf{X}^{T} \mathbf{X}\right)^{-1} \Delta \mathbf{X}^{T},
\end{aligned}
$$

using the relation $\Delta\left[\mathbf{Y}^{-1}\right]=-\mathbf{Y}^{-1} \Delta[\mathbf{Y}] \mathbf{Y}^{-1}$ for invertible matrix $\mathbf{Y}=\mathbf{X}^{T} \mathbf{X}$. Then, the vectorization of $\Delta \mathbf{X}^{\dagger}$ is obtained as

$$
\operatorname{vec}\left\{\Delta \mathbf{X}^{\dagger}\right\}=-\left[\left(\mathbf{X X}^{\dagger}\right)^{T} \otimes\left(\mathbf{X}^{T} \mathbf{X}\right)^{-1}\right] \operatorname{vec}\left\{\Delta \mathbf{X}^{T}\right\}-\left[\left(\mathbf{X}^{\dagger}\right)^{T} \otimes \mathbf{X}^{\dagger}\right] \operatorname{vec}\{\Delta \mathbf{X}\}+\left[\mathbf{I}_{a} \otimes\left(\mathbf{X}^{T} \mathbf{X}\right)^{-1}\right] \operatorname{vec}\left\{\Delta \mathbf{X}^{T}\right\}
$$

Define the permutation matrix $\mathcal{P}_{a, b}$ such that $[9,10]$

$$
\operatorname{vec}\left\{\mathbf{X}^{T}\right\}=\mathcal{P}_{a, b} \operatorname{vec}\{\mathbf{X}\}
$$

Hence:

$$
\operatorname{vec}\left\{\Delta \mathbf{X}^{\dagger}\right\}=\left(\left\{-\left[\left(\mathbf{X} \mathbf{X}^{\dagger}\right)^{T} \otimes\left(\mathbf{X}^{T} \mathbf{X}\right)^{-1}\right]+\left[\mathbf{I}_{a} \otimes\left(\mathbf{X}^{T} \mathbf{X}\right)^{-1}\right]\right\} \mathcal{P}_{a, b}-\left[\left(\mathbf{X}^{\dagger}\right)^{T} \otimes \mathbf{X}^{\dagger}\right]\right) \operatorname{vec}\{\Delta \mathbf{X}\}
$$

Appendix B. Perturbation of $\mathbf{Y}=\left[\mathbf{I}_{2 n_{r}} \otimes\left(\mathbf{I}_{(p+1) r}-\hat{O} \hat{O}^{\dagger}\right)\right] \operatorname{vec}\{\mathbf{X}\}$

Consider the relation

$$
\mathbf{Y}=\left[\mathbf{I}_{2 n_{r}} \otimes\left(\mathbf{I}_{(p+1) r}-\hat{O} \hat{O}^{\dagger}\right)\right] \operatorname{vec}\{\mathbf{X}\}
$$

where $\mathbf{X} \in \mathbb{R}^{(p+1) r \times 2 n_{r}}, \hat{O}$ and $\mathbf{X}$ are afflicted with uncertainties, and assume

$$
\operatorname{vec}\{\Delta \mathbf{X}\}=\mathcal{J}_{\mathbf{X}, \theta_{k-1}^{h}} \Delta \boldsymbol{\theta}_{k-1}^{h}
$$

The goal is to derive the relationship between $\operatorname{vec}\{\Delta \mathbf{Y}\}$ and $\operatorname{vec}\{\Delta \hat{O}\}$.

From (B.1) it follows

$$
\Delta \mathbf{Y}=-\left[\mathbf{I}_{2 \mathbf{n}_{\mathbf{r}}} \otimes\left(\Delta\left[\hat{O} \hat{O}^{\dagger}\right]\right)\right] \operatorname{vec}\{\mathbf{X}\}+\left[\mathbf{I}_{2 \mathbf{n}_{\mathbf{r}}} \otimes\left(\mathbf{I}_{(\mathbf{p}+\mathbf{1}) \mathbf{r}}-\hat{O} \hat{O}^{\dagger}\right)\right] \operatorname{vec}\{\boldsymbol{\Delta} \mathbf{X}\}
$$


- The first term in Eq. (B.3) can be developed as

$$
\left[\mathbf{I}_{2 n_{r}} \otimes\left(\Delta\left[\hat{O} \hat{O}^{\dagger}\right]\right)\right] \operatorname{vec}\{\mathbf{X}\}=\left[\mathbf{I}_{2 n_{r}} \otimes \Delta \hat{O} \hat{O}^{\dagger}\right] \operatorname{vec}\{\mathbf{X}\}+\left[\mathbf{I}_{2 n_{r}} \otimes \hat{O} \Delta \hat{O}^{\dagger}\right] \operatorname{vec}\{\mathbf{X}\}
$$

where

- by using the relation $\left(\mathbf{I}_{2 n_{r}} \otimes \mathbf{A B}\right) \operatorname{vec}\{\mathbf{C}\}=\left((\mathbf{B C})^{T} \otimes \mathbf{I}_{(p+1) r}\right) \operatorname{vec}\{\mathbf{A}\}$, where $\mathbf{A} \in \mathbb{R}^{(p+1) r \times 2 n}, \mathbf{B} \in \mathbb{R}^{2 n \times(p+1) r}$ and $\mathbf{C} \in \mathbb{R}^{(p+1) r \times 2 n_{r}}[26]$

$$
\left[\mathbf{I}_{2 n_{r}} \otimes \Delta \hat{O} \hat{O}^{\dagger}\right] \operatorname{vec}\{\mathbf{X}\}=\left[\left(\hat{O}^{\dagger} \mathbf{X}\right)^{T} \otimes \mathbf{I}_{(p+1) r}\right] \operatorname{vec}\{\Delta \hat{O}\}
$$

- by using the relation $\left(\mathbf{I}_{2 n_{r}} \otimes \mathbf{A B}\right) \operatorname{vec}\{\mathbf{C}\}=\left(\mathbf{C}^{T} \otimes \mathbf{A}\right) \operatorname{vec}\{\mathbf{B}\}$

$$
\left[\mathbf{I}_{2 n_{r}} \otimes \hat{O} \Delta \hat{O}^{\dagger}\right] \operatorname{vec}\{\mathbf{X}\}=\left(\mathbf{X}^{T} \otimes \hat{O}\right) \operatorname{vec}\left\{\Delta \hat{O}^{\dagger}\right\}
$$

From Eq. (A.4) it comes that

$$
\operatorname{vec}\left\{\Delta \hat{O}^{\dagger}\right\}=\mathcal{K} \operatorname{vec}\{\Delta \hat{O}\}
$$

where

$$
\mathcal{K}=\left\{-\left[\left(\hat{O} \hat{O}^{\dagger}\right)^{T} \otimes\left(\hat{O}^{T} \hat{O}\right)^{-1}\right]+\left[\mathbf{I}_{(p+1) r} \otimes\left(\hat{O}^{T} \hat{O}\right)^{-1}\right]\right\} \mathcal{P}_{(p+1) r, 2 n}-\left[\left(\hat{O}^{\dagger}\right)^{T} \otimes \hat{O}^{\dagger}\right]
$$

Eq. (B.6) is then formulated as

$$
\left[\mathbf{I}_{2 n_{r}} \otimes \hat{O} \Delta \hat{O}^{\dagger}\right] \operatorname{vec}\{\mathbf{X}\}=\left(\mathbf{X}^{T} \otimes \hat{O}\right) \mathcal{K} \operatorname{vec}\{\Delta \hat{O}\}
$$

- Using Eq. (B.2), the second term in Eq. (B.3) can be developed as

$$
\left[\mathbf{I}_{2 n_{r}} \otimes\left(\mathbf{I}_{(p+1) r}-\hat{O} \hat{O}^{\dagger}\right)\right] \operatorname{vec}\{\Delta \mathbf{X}\}=\left[\mathbf{I}_{2 n_{r}} \otimes\left(\mathbf{I}_{(p+1) r}-\hat{O} \hat{O}^{\dagger}\right)\right] \mathcal{J}_{\mathbf{X}, \theta_{k-1}^{h}} \Delta \boldsymbol{\theta}_{k-1}^{h} .
$$

By using Eq. (47), i.e.,

$$
\Delta \boldsymbol{\theta}_{k-1}^{h}=\mathcal{M}_{k-1} \operatorname{vec}\{\Delta \hat{O}\}
$$

Eq. (B.10) is rewritten as

$$
\left[\mathbf{I}_{2 n_{r}} \otimes\left(\mathbf{I}_{(p+1) r}-\hat{O} \hat{O}^{\dagger}\right)\right] \operatorname{vec}\{\Delta \mathbf{X}\}=\left[\mathbf{I}_{2 n_{r}} \otimes\left(\mathbf{I}_{(p+1) r}-\hat{O} \hat{O}^{\dagger}\right)\right] \mathcal{J}_{\mathbf{X}, \theta_{k-1}^{h}} \mathcal{M}_{k-1} \operatorname{vec}\{\Delta \hat{O}\}
$$

By introducing Eqs. (B.4), (B.5), (B.9) and (B.12) in Eq. (B.3), the relation between $\operatorname{vec}\{\Delta \mathbf{Y}\}$ and $\operatorname{vec}\{\Delta \hat{O}\}$ writes as

$$
\Delta \mathbf{Y}=\mathcal{J}_{\mathbf{Y}, \hat{O}} \operatorname{vec}\{\Delta \hat{O}\}
$$

where

$$
\mathcal{J}_{\mathbf{Y}, \hat{O}}=-\left[\left(\hat{O}^{\dagger} \mathbf{X}\right)^{T} \otimes \mathbf{I}_{(p+1) r}\right]-\left[\mathbf{X}^{T} \otimes \hat{O}\right] \mathcal{K}+\left[\mathbf{I}_{2 n_{r}} \otimes\left(\mathbf{I}_{(p+1) r}-\hat{O} \hat{O}^{\dagger}\right)\right] \mathcal{J}_{\mathbf{X}, \boldsymbol{\theta}_{k-1}^{h}} \mathcal{M}_{k-1}
$$




\section{References}

[1] W. Heylen, S. Lammens, P. Sas, Modal Analysis Theory and Testing, Katholieke Universiteit Leuven, Belgium, 1998.

[2] N. Maia, J. Silva, Modal analysis identification techniques, Philosophical Transactions of the Royal Society of London A: Mathematical, Physical and Engineering Sciences 359 (1778) (2001) 29-40.

[3] B. Peeters, G. De Roeck, Stochastic system identification for operational modal analysis: a review, Journal of Dynamic Systems, Measurement, and Control 123 (4) (2001) 659-667.

[4] P. Van Overschee, B. De Moor, Subspace Identification for Linear Systems: Theory, Implementation, Applications, Kluwer, 1996.

[5] B. Peeters, G. De Roeck, Reference-based stochastic subspace identification for output-only modal analysis, Mechanical systems and signal processing 13 (6) (1999) 855-878.

[6] A. Benveniste, L. Mevel, Nonstationary consistency of subspace methods, IEEE Transactions on Automatic Control AC-52 (6) (2007) 974984.

[7] M. Döhler, L. Mevel, Fast multi-order computation of system matrices in subspace-based system identification, Control Engineering Practice 20 (9) (2012) 882-894.

[8] R. Pintelon, P. Guillaume, J. Schoukens, Uncertainty calculation in (operational) modal analysis, Mechanical systems and signal processing 21 (6) (2007) 2359-2373.

[9] E. Reynders, R. Pintelon, G. De Roeck, Uncertainty bounds on modal parameters obtained from stochastic subspace identification, Mechanical Systems and Signal Processing 22 (4) (2008) 948-969.

[10] M. Döhler, L. Mevel, Efficient multi-order uncertainty computation for stochastic subspace identification, Mechanical Systems and Signal Processing 38 (2) (2013) 346-366

[11] M. Friswell, Damage identification using inverse methods, Philosophical Transactions of the Royal Society A: Mathematical, Physical and Engineering Sciences 365 (1851) (2007) 393-410.

[12] E. Simoen, G. De Roeck, G. Lombaert, Dealing with uncertainty in model updating for damage assessment: A review, Mechanical Systems and Signal Processing 56 (2015) 123-149.

[13] M. Friswell, J. E. Mottershead, Finite element model updating in structural dynamics, Vol. 38, Kluwer, 1995.

[14] J. E. Mottershead, M. Link, M. I. Friswell, The sensitivity method in finite element model updating: a tutorial, Mechanical systems and signal processing 25 (7) (2011) 2275-2296.

[15] A. Der Kiureghian, O. Ditlevsen, Aleatory or epistemic? does it matter?, Structural Safety 31 (2) (2009) $105-112$.

[16] H. H. Khodaparast, J. E. Mottershead, M. I. Friswell, Perturbation methods for the estimation of parameter variability in stochastic model updating, Mechanical Systems and Signal Processing 22 (8) (2008) 1751-1773.

[17] G. Gautier, J.-M. Mencik, R. Serra, A finite element-based subspace fitting approach for structure identification and damage localization, Mechanical Systems and Signal Processing 58-59 (0) (2015) 143 - 159.

[18] A. Swindlehust, R. Roy, B. Ottersten, T. Kailath, A subspace fitting method for identification of linear state-space models, Automatic Control, IEEE Transactions on 40 (2) (1995) 311-316.

[19] G. Tondreau, A. Deraemaeker, Numerical and experimental analysis of uncertainty on modal parameters estimated with the stochastic subspace method, Journal of sound and vibration 333 (18) (2014) 4376-4401.

[20] P. Stoica, R. L. Moses, Introduction to spectral analysis, Vol. 1, 1997.

[21] G. Golub, V. Pereyra, Separable nonlinear least squares: the variable projection method and its applications, Inverse problems 19 (2) (2003) R1.

[22] Y. Wang, Gauss-newton method, Wiley Interdisciplinary Reviews: Computational Statistics 4 (4) (2012) $415-420$.

[23] P. Andersen, Estimation of modal parameters and their uncertainties, Tech. rep., Dept. of Building Technology and Structural Engineering, Aalborg University (1998).

[24] E. Hannan, Multiple time series (wiley series in probability and mathematical statistics)

[25] G. Casella, R. L. Berger, Statistical inference, thomson learning, Duxbury Press: Belmont, CA. 
[26] J. W. Brewer, Kronecker products and matrix calculus in system theory, Circuits and Systems, IEEE Transactions on 25 (9) (1978) $772-781$.

[27] W. Gawronski, Discrete-time norms of flexible structures, Journal of Sound and Vibration 264 (2003) 983-1004.

[28] C. Z. Mooney, Monte carlo simulation, Vol. 116, Sage Publications, 1997.

[29] D. Bauer, M. Deistler, W. Scherrer, Consistency and asymptotic normality of some subspace algorithms for systems without observed inputs, Automatica 35 (7) (1999) 1243-1254. 